\title{
DIE ZWEITE PRIVILEGURKUNDE FÜR DIE PEGAI-MONEMBASIOTEN - EINE FÄLSCHUNG?*
}

Guillaume de Villehardouin (reg. ab 1246) gelang es ca.1250 nach mehrjähriger Belagerung das bis dahin griechische Monembasia in Besitz zu nehmen ${ }^{1}$, unter der vertraglichen Verpflichtung, den Einwohnern, wenn sie

* Für ihre kritische Durchsicht des Typoskriptes, woraus etliche Ergänzungen und Korrekturen resultierten, hat der Verfasser zu danken Doz. K. Belke/ Wien, Prof. O. Kresten / Wien, Prof. K.-P. Matschke / Leipzig und Prof. P. Schreiner / Köln.

${ }^{1}$ Die Chronik von Morea, ed. J. Sснмптт. London 1904, vv. 2756-2790, 2901-2959, gibt v. 2930 die Belagerungsdauer mit drei Jahren und mehr an, was für 1250 spricht, als der Fürst vom Kreuzzug von Ludwig IX. (J. Longnon, L'empire latin de Constantinople et la principauté de Morée. Paris 1949, 218-219) zurückgekehrt war; die Präsenz von Guillaume im Raum von Lakedaimonia im Winter 1248/49 ist kein Indiz für eine bereits erfolgte Einnahme von Monembasia, wie Longnon, op. cit. 216 meint. Die Anaphora B

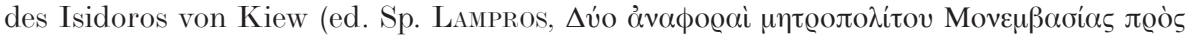

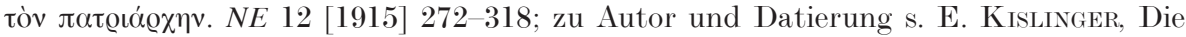
Sizilienfahrt des Isidoros von Kiev [142]. Diptycha 6 [1994/95] 49-65, hier 59-64) spricht von sieben Jahren Belagerung durch Guillaume, was von dessen Herrschaftsbeginn 1246 an das Jahr 1253 ergeben würde. Isidoros verwechselt dabei allerdings - trotz des richtig erkannten Konnexes mit Leon Sguros (wahrscheinlich auch auf Basis von Niketas Choniates 605, 61-73, 611, 26-35 [VAN DieTen]) - Guillaume (287, 27-31 [LAMpros]) mit Geoffroy de Villehardouin, unter dem 1210/1212 Korinth und Nauplion erobert wurden (A. Bon, La Morée franque. Recherches historiques, topographiques et archéologiques sur la principauté d’Achaie [1205-1430]. Paris 1969, I 64-73; M. S. Kondoses,

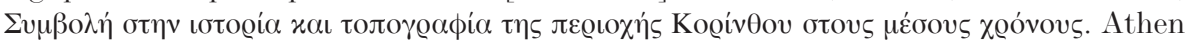
1981, 95-102). Möglicherweise fußt der Irrtum des Isidoros eben auf der Chronik von Morea oder mündlicher Tradition (die auch dort einfloß), welche die Einnahme von Korinth und Nauplion erst Guillaume zuschreibt und dem Fall von Monembasia vorangehen läßt. Zuerst galt es, Verbündete wie Venedig zu finden (vv. 2778-2800 [Sснмгтт]), dann fiel Korinth in einer Kampagne (vv. 2801-2827 [Sснмгтт], während Nauplion zwei Jahre widerstand [vv. 2860-2874 [Sснмгтт]). Zusammen kann Isidoros daraus auf rechnerisch sieben Jahre seit dem grundsätzlichen Beschluß gekommen sein, was nebenbei seiner eigenen Aussage widerspricht (288, 11 [LAMpros]), nach Korinth

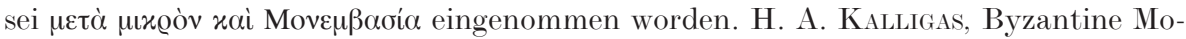
nemvasia. The Sources. Monemvasia 1990, 86-94 vermerkt allerdings 91 zurecht, erst 1253 sei ein lateinischer Bischof für Monembasia gewählt worden. 
dies wünschten, freien Abzug zu gestatten². Wer diese Wahl traf ${ }^{3}$, wurde vom nikänischen Kaiserreich ${ }^{4}$ zu Pegai (heute Karabiga, 56 km westlich von Bandirma) an der kleinasiatischen Küste des Marmarameeres angesiedelt $^{5}$. Auch in der neuen Heimat lebten die Monembasioten offenbar von Handel zur See ${ }^{6}$.

Zeugnis dafür legen zwei Privilegurkunden ab (ein Prostagma bzw. ein Chrysobull, im folgenden als P[egai] I und P II bezeichnet), die beide literarisch überliefert sind und gemeinsam kritisch ediert wurden ${ }^{7}$. Der Herausgeber, P. Schreiner, hat ihre Wertigkeit jedoch ganz unterschiedlich beurteilt:

2 Anaphora B des Isidoros 289,17-18 (Lampros). Von einer Übereinkunft vor Übergabe der Stadt spricht auch die Chronik von Morea, vv. 2936-2941 (Хснмгтт), ohne freilich die Möglichkeit des freien Abzuges zu erwähnen. Kaldigas, Monemvasia 89: “The important differences in the information given by each source must be related to the effort of each chronicler to present the events under a more favourable light for his side".

${ }^{3}$ Der Exodus war nicht umfassend, es verblieben laut der Chronik von Morea vv. 29412955 (Sснмгтт) die führenden Familien (Mamonas, Daimonogiannes und Sophianos), wozu die Aussage der Anaphora B des Isidoros 290,5-15 (Lampros) paßt, auch der Bischof habe trotz schwieriger Bedingungen in Monembasia ausgeharrt.

${ }^{4} \mathrm{Zu}$ früheren Kontakten von Monembasia dorthin vgl. M. S. Kordoses, Southern Greece under the Franks (1204-1262). A Study of the Greek Population and the Orthodox Church under Frankish Domination. Ioannina 1987, 29-32, 88-91.

5 Anaphora B des Isidoros 289, 27-29 (Lampros). Zum Ort F. W. Hasluck, Cyzicus. Cambridge 1910, 98-100 (mit Karte); ODB III 1615-1616.

${ }^{6}$ A. Laiou, The Byzantine Economy in the Mediterranean Trade System; ThirteenthFifteenth Centuries. DOP 34/35 (1980/81) 177-222, v.a. 205-208 (Reprint in DIES., Gender, Society and Economic Life in Byzantium. London 1992, Nr. VII); D. ZакутнIvos, Le Despotat grec de Morée. Vie et institutions. London ${ }^{2} 1975,255-256$; Kaldigas,

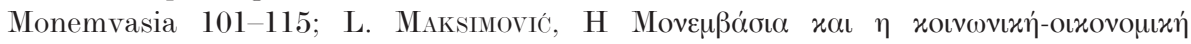

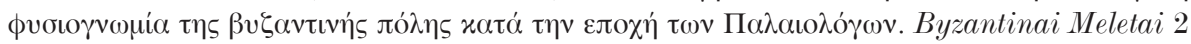

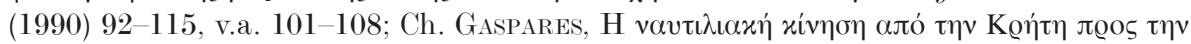

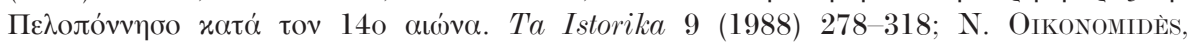
Hommes d'affaires grecs et latins à Constantinople (XIIIe-X Ve siècles). Montreal-Paris 1979, 87-88; K.-P. Matschke, Byzantinische Politiker und byzantinische Kaufleute im Ringen um die Beteiligung am Schwarzmeerhandel in der Mitte des 14. Jahrhunderts. Mitteilungen des bulgarischen Forschungsinstitutes in Österreich 2/VI (1984) 75-96, hier 83-87; DERs., Bemerkungen zu den sozialen Trägern des spätbyzantinischen Seehandels. Byzantino-Bulgarica 7 (1981) 253-261, hier 255; G. Makris, Studien zur spätbyzantinischen Schiffahrt (Collana storica di fonti e studi 52). Genua 1988, 70-71. Zur mitunter alternativ betriebenen Piraterie s. G. Morgan, The Venetian Claims Commission of 1278. BZ 69 (1976) 411-438.

${ }^{7}$ P. Schreiner, Ein Prostagma Andronikos III. für die Monembasioten in Pegai (1328) und das gefälschte Chrysobull Andronikos II. für die Monembasioten im byzantinischen Reich. JÖB 27 (1978) 203-228, ebendort 207-213 Edition von P I, 217-225 von P II (hier wiederholt 225-227). 
Bei der in Codex Vat. gr. 2228 (S. XIV), f. 314-315 überlieferten Urkunde P I, welche man (aufgrund vor allem eigener Angaben ${ }^{8}$ ) Andronikos III. zuzuweisen hat und die 1328 erging $^{9}$, ,ist an der Glaubwürdigkeit des Dokuments nicht zu zweifeln"10. Das Prostagma erneuert und präzisiert

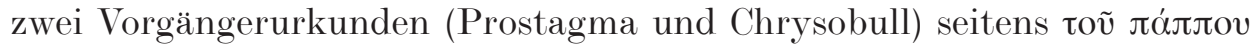

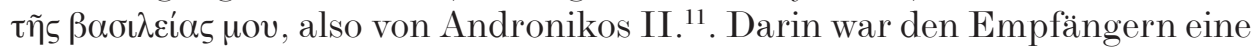
Zollreduktion zu Konstantinopel und in den „makedonischen“ Städten (so Herakleia [Marmara Ereğlisi], Selymbria [Silivri], Rhaidestos [Tekirdağ], Kallipolis [Gelibolu]) am Marmarameer ${ }^{12}$ gewährt worden (207, Z. 4-6

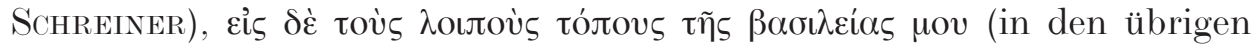
Orten meines Reiches) sollten sie überhaupt keinen Zoll und sonstige Abgaben zahlen (Z. 6-9). Erstere Konzession wird nun bestätigend wiederholt (207, Z. 10-18) ${ }^{13}$, wobei Ainos (Enez) zusätzlich genannt wird (Z. 15) $)^{14}$,

${ }^{8}$ Es sind dies die Aussage, daß der (ausstellende) Kaiser verheiratet sei und seine Mutter (noch) lebe (211, Z. 44-45 Schreiner), sowie die Datierung auf August in einer 11. Indiktion (213, Z. 58), die - ergänzt um einen postulierten Verlust von Pegai an die Osmanen spätestens 1340 (dazu allerdings unten 215-216) - gemeinsam nur auf Andronikos III. anno 1328 zutreffen (PLP VIII Nr. 21437). Vgl. im Einzelnen die überzeugende Darlegung bei Schreiner, Prostagma 204-205.

${ }^{9}$ In F. Dölger, Regesten der Kaiserurkunden des oströmischen Reiches von 565-1453, 4. Teil: Regesten von 1282-1341. München-Berlin 1960, fehlt, da die Urkunde zur Abfassungszeit des Regestenbandes noch unbekannt war, ein diesbezügliches Regest, Nr. 2383 bezieht sich auf P II.

10 Schreiner, Prostagma 203.

11 PLP VIII Nr. 21436. Zum Chrysobull (unzutreffend [bedingt durch P II] für Monembasia) s. F. DöLger, Regesten der Kaiserurkunden des oströmischen Reiches von 5651453, 3. Teil: Regesten von 1204-1282. Zweite, erweiterte und verbesserte Auflage, bearbeitet von P. Wirth. München 1977, Nr. 1897; ein Regest zum Prostagma fehlt.

${ }_{12} \mathrm{Zu}$ Herakleia ist im späten 13. Jahrhundert eine monembasiotische Kolonie bezeugt: Gregorios Kyprios, Brief 149 (141-142 Eustratiades) (= Brief 154 nach W. Lameere, La tradition manuscrite de la correspondance de Grégoire de Chypre, patriarche de Constantinople [1283-1289]. Brüssel-Rom 1937). Мatschke, Byzantinische Politiker 88

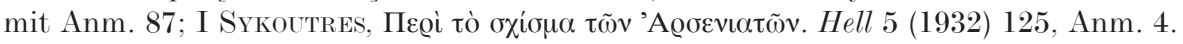

${ }^{13}$ Hiermit erledigt sich die Behauptung von Kaldigas, Monemvasia 120, daß die PegaiMonembasioten 1328 lediglich ersucht hätten, die ihnen erlassenen Abgaben zu spezifizieren. Der erst kurz zuvor im Mai erfolgte Regierungswechsel lieferte vielmehr den Anlaß, vom neuen Herrscher die Bestätigung des alten Privilegs insgesamt seines Vorgängers zu erbitten.

${ }^{14}$ P. Soustal, Thrakien (Thrake, Rhodope und Haimimontos) (TIB 6). Wien 1991, 170 173; zu seiner wirtschaftlichen Bedeutung s. K.-P. Matschke, The Late Byzantine Urban Economy, Thirteenth-Fifteenth Centuries, in: Economic History of Byzantium, ed. A.E. Laiou, Ch. Bouras, C. Morrisson, N. Oikonomides, K. Pitsakis. (Internet-Preprint) Washington, D.C. 2002, 455-487, hier 460, 466; C. Asdracha, La région des Rhodopes aux XIIIe et XIVe siècles. Etude de géographie historique (Texte und Forschungen zur Byzantinisch-Neugriechischen Philologie 49). Athen 1976, 120-124, 193-196. 
sodann erfahren die sonstigen Abgaben eine detaillierte Auflistung (209, Z. 19-23). Die involvierten Handelspartner der Begünstigten (Käufer, Verkäufer und die Waren transportierende Schiffseigner) haben bei deren Geschäften überhaupt keine Abgaben zu leisten (Z. 23-26). Diesbezüglich

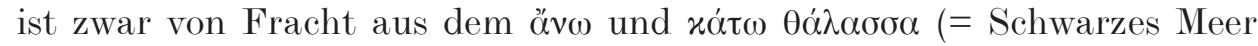
bzw. Ägäis) die Rede (211, Z. 27-28) ${ }^{15}$, aber schon der Bezug auf abgabenfreien Transit im „makedonischen“ Raum bestätigt (Z. 32-35) erneut den Eindruck, daß die Marmarameer-Region, 1328 verstärkt deren europäische, noch ganz byzantinisch kontrollierte Seite, samt ihrem thrakischen Hinterland das Haupthandelsgebiet ${ }^{16}$ der Pegai-Monembasioten bildete. Das Staat hob deswegen hier - wenngleich reduzierte - Abgabensätze ein,

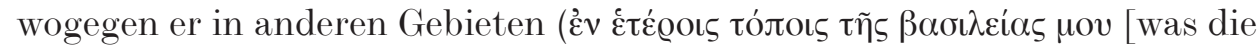
Formulierung des früheren Prostagmas/Chrysobulls aufgreift] „im Osten, im Westen, auf den Inseln" [Z. 36-37] ob des geringen Volumens leicht darauf verzichten konnte.

Das Chrysobull P II (die folgenden Zeilenangaben daraus beziehen sich auf den bis auf die Seitenzahlen unveränderten Wiederabdruck hier im Anhang 225-227 der Edition von Schreiner) gibt an, von Andronikos II. zu stammen (Z. 80-84 $)^{17}$, könnte daher theoretisch das entsprechende Vorgängerdokument laut P I (dort Z. 2-3) sein. Aus dem Wortlaut wird allerdings klar, daß ganz im Gegenteil das Prostagma P I die textliche Grundlage für P II bot ${ }^{18}$, immer wieder mit eingefügten Ergänzungen (in der Edition kursiv gesetzt). Zum einen gelten sie einer weiteren Zollsenkung (Z. 22-25)

15 Für monembasiotische Händler im Bereich des Schwarzen Meeres s. LaIou, Economy 206 mit Anm. 115; K.-P. Matschke, Commerce, Trade, Markets and Money: ThirteenthFifteenth Centuries, in: Economic History of Byzantium (wie Anm. 14) 763-798, hier 782; DERs., Byzantinische Politiker 84-86, 88. Aus dem Ägäisraum wird höchstwahrscheinlich der Monembasia-Wein (Malvasier) eine bevorzugte Handelsware gewesen sein (Zakythinos, Despotat II 249-250). Kaufleute aus Monembasia sind 1341-1344 in Konstantinopel anwesend und überbringen ihrem designierten Metropoliten, dem späteren Patriarchen Isidoros, dreihundert Hyperpyra: Leben des Isidoros, ed. D. G.

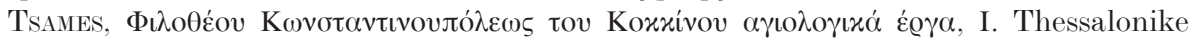
$1985,365-368$ (cap. 31, 34).

${ }^{16}$ Die wichtigsten Güter waren Getreide, Wein, eingepökelte Lebensmittel, Schlachtvieh, Holz und Wollstoffe (207, Z. 15-17, 211, Z. 33). Aufgrund der starken Konkurrenz westlicher Händler im gehobenen Textilsektor wird es sich bei letzteren um einfache Produkte und zuliefernden Aufkauf im ländlichen Raum gehandelt haben, vgl. МatschKE, Commerce 763-764, 768-769 und speziell 777 (Ankäufe des Giacomo Badoer von Rhaidestos aus mittels griechischer Agenten).

17 Dölger, Regesten IV Nr. 2383, der noch in Unkenntnis von P II die Datierung für richtig erachtet (anders zuvor in BZ 34 [1934] 126).

18 Schreiner, Prostagma 215. 
und neuen Abgabenbefreiungen (Z. 30-38), zum anderen wird der Kreis der Nutznießer wesentlich erweitert: Zu den Pegai-Monembasioten treten jetzt ebenso die Bewohner von Monembasia selbst (und überhaupt alle im Reich lebenden Monembasioten) (Z. 1-4, 15-22). Die Quelle, welche uns das Chrysobull überliefert, nämlich das Chronicon maius des Georgios Sphrantzes ${ }^{19}$, legt bereits nahe, wer tatsächlich für die besagten Abänderungen verantwortlich zeichnet. Gegenüber dem originalen Sphrantzes-Text (Chronicon minus) ${ }^{20}$ enthält die Maius-Fassung zahlreiche Interpolationen seitens des Makarios Melissenos ${ }^{21}$. Dieser Oberhirte von Monembasia (1569?-1571) „hat aus dem Prostagma für die kleine monembasiotische Gemeinde in Pegai durch Erweiterungen und Zusätze ein Chrysobull zugunsten seiner Bischofsstadt gemacht, das de facto im 16. Jahrhundert natürlich wertlos war, die ruhmreiche Vergangenheit seines Sitzes aber betonen sollte“22.

Besonderes Geschick läßt Makarios Melissenos dabei vermissen. Zuviel seines Eifers ist es, die Abgabenfreiheit an allen Orten der Peloponnes zu vermerken (Z. 57-61), da sie (auf Basis von P I) ohnehin zuvor bereits für alle Reichsgebiete außerhalb der Marmara-Meer-Region gewährt worden war (Z. 55-57) $)^{23}$. Mehrmals wird sprachliches Unverständnis des Urtextes

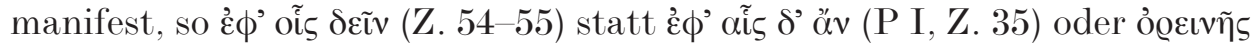

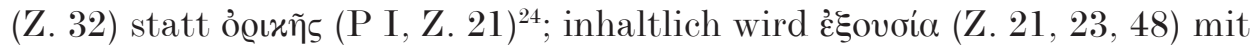

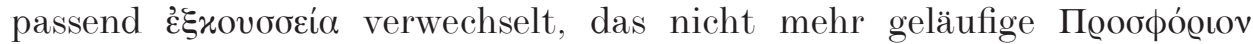

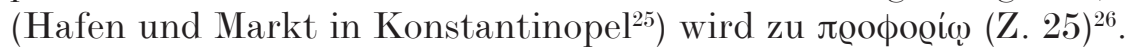

19 Ed. V. Grecu, Georgios Sphrantzes, Memorii 1401-1477. Bukarest 1976, 538-542 (Pseudo-Phrantzes: Macarie Melissenos, Cronica 1258-1481). H. Hunger, Die hochsprachliche profane Literatur der Byzantiner, I (Handb. d. Altertumswiss. XII 5/1). München $1978,494-499$.

${ }^{20}$ Ed. R. Maisano, Giorgio Sfranze, Cronaca (CFHB XXIX). Rom 1990.

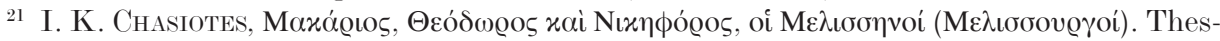
salonike 1966, 23-26, 171-182; R.-J. LoENerTZ, Autour du chronicon maius attribué à Georges Phrantzès, in: Miscellanea G. Mercati III. Vatikanstadt 1946, 273-311; F. Dölger, Ein literarischer und diplomatischer Fälscher des 16. Jahrhunderts: Metropolit Makarios von Monembasia, in: F. Dölger, Byzantinische Diplomatik. Ettal 1956, 371-383; V. Grecu, Georgios Sphrantzes, Leben und Werk. Makarios Melissenos und sein Werk. Die Ausgabe. BSl 26 (1965) 62-73.

22 Schreiner, Prostagma 215.

${ }^{23}$ Vgl. bereits Schreiner, Prostagma 223 (Kommentar zu Z. 57): „Aus dem Einschub spricht der Patriotismus des Peloponnesiers Makarios Melissenos“.

24 Schreiner, Prostagma 219, 222.

${ }^{25}$ R. Janin, Constantinople byzantine. Développement urbain et répertoire topographique. Paris 1964, 235-236; A. Berger, Untersuchungen zu den Patria Konstantinupoleos (Poikila Byzantina 8). Bonn 1988, 424-426; W. Múller-Wiener, Die Häfen von Byzantion - Konstantinupolis - Istanbul. Tübingen 1994, 4, 13.

${ }^{26}$ Schreiner, Prostagma 217, 218. 
Essentiell und entlarvend ist aber doch das vorgebliche Ergehen unter Andronikos II., wo doch, wie erwähnt, die zugrundeliegende Textbasis (P I) erst unter seinem Nachfolger entstand. Dem nicht genug, war die kaiserliche Unterschrift in dieser Form zum angeblichen Ausstellungsdatum (Z. 80-81: 15. Indiktion, November des Jahres $6825=1316$ AD) gar nicht mehr üblich ${ }^{27}$, sie wurde aus irgendeinem älteren Dokument mechanisch kopierend übernommen. Makarios widerspricht sich sogar selbst, wenn er einleitend zum Dokument behauptet, es stamme von Andronikos III. ${ }^{28}$ Die Indizienlage wirkt erdrückend, welche den Editor zum abschließenden Urteil brachte: „Keinem einzigen Teil dieser Urkunde kann auch nur in irgendeiner Form historische Beweiskraft zukommen “29.

Einen solch umfassenden „Schuldspruch“ akzeptierte die Fachmeinung keineswegs einhellig ${ }^{30}$. Anlaß zur Skepsis bieten mehrere in P II modifizierte/ ergänzte Passagen, die mit den manipulativen Absichten des Makarios im 16. Jahrhundert logisch unvereinbar sind.

Am Beginn des Chrysobulls erfahren wir, die aus Pegai stammenden

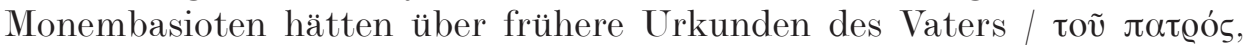

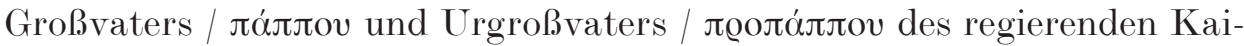
sers verfügt (Z. 4-6 ${ }^{31}$; P I sprach dagegen Z. 2 nur von einem Prostagma und einem Chrysobull von Andronikos II., des Großvaters von Andronikos III.). Sofern Makarios die Abänderung vornahm, gibt es zwei Möglichkeiten, je nachdem welchem Kaiser er das Chrysobull zuschreiben wollte:

a) Im Falle von Andronikos II., zu dem Datierung und prinzipiell (s. oben) die Unterschrift passen, wären die Vorgängerdokumente von Michael VIII. (Vater), Andronikos Palaiologos (Großvater) und Alexios Palaiologos (Urgroßvater) ausgestellt worden. Das ist schon beim Großvater ausgeschlossen, da er als Megas Domestikos kein kaiserliches Chrysobull

${ }^{27}$ F. Dölger in BZ 34 (1934) 126, Anm. 1; D. I. Polemis, The Doukai. A Contribution to Byzantine Prosopography. London 1968, 158.

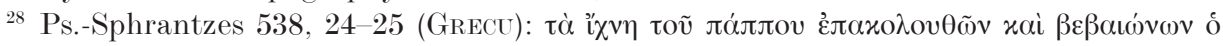

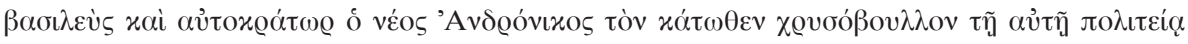

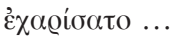

29 Schreiner, Prostagma 215.

30 Zustimmend Maksimović, Moveußaбía 106-107 und Makris, Schiffahrt 70-71, Anm. 13. Zweifel äußerten Laiou, Economy 207, Oikonomidès, Hommes d'affaires 88 und KaLLIGAS, Monemvasia 118-119, 129-133.

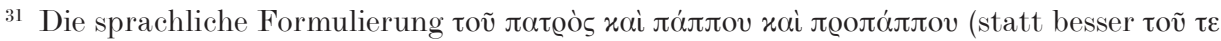

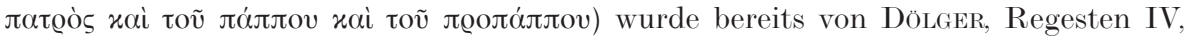
Nr. 2383 als Indiz einer Verfälschung seitens Makarios aufgefaßt (ihm folgt ScHreiner, Prostagma 217, Kommentar zu Z. 6), eine sprachliche Verschlechterung muß aber nicht, wie oben gezeigt, den Wortlaut insgesamt in Frage stellen. 
erlassen konnte, ganz abgesehen davon, daß bis zu seinem Tod 1247 noch gar keine monembasiotische Kolonie in Pegai bestand ${ }^{32}$.

b) Wenn doch Andronikos III. als Aussteller von P II gemeint war (wie Makarios einleitend behauptete, s. oben 210 mit Anm. 28), stammen die früheren Privilegien von Michael IX.33, Andronikos II. und Michael VIII. ${ }^{34}$, was für Andronikos II. belegt bzw. bei den anderen beiden durchaus denkbar ist ${ }^{35}$.

Sofern man Makarios nicht unterstellen will, daß er völlig unfähig war, die genealogischen Verhältnisse der Palaiologenherrscher zu erfassen, bleibt als a) und b) gerechtwerdender Kompromiß nur, daß der Oberhirte kein Dokument von Andronikos III. verfügbar hatte, welches er für die in P II erforderliche Datierung und Unterschrift heranziehen konnte. Er griff deshalb wider besseres Wissen auf Andronikos II. zurück ${ }^{36}$, dessen echtes Chrysobull für die Metropolis von Monembasia von 1301 (Fassung B) er ja bereits verfälscht hatte (Fassung A) ${ }^{37}$.

Bei einer Fälschung von P II sozusagen aus einem Guß über zwei Jahrhunderte später wäre es schwerlich von Relevanz gewesen, die in P I vorhandene Erwähnung der Kaiserinmutter (Z. 45) wegzulassen, wie dies geschieht. Rita von Armenien (Maria Dukaina Palaiologina) ${ }^{38}$ ist Juni/Juli 1333 verstorben, die hingegen in P II (Z. 65) gleich P I (Z. 45 [211 SchreINER]) vertretene Gattin des Andronikos III., Johanna von Savoyen (Anna Palaiologina) ${ }^{39}$, hat bekanntlich sogar den Kaiser (gest. 15. 6. 1341) überlebt. Gewiß mag hier mit einer versehentlichen Auslassung argumentiert werden, aber ein derart resultierendes Ausstellungsdatum nach Juli 1333

32 J.-F. VAnnier, Les premiers Paléologues. Etude généalogique et prosopographique, in: Etudes prosopographiques, par J.-C. Cheynet - J.-F. VANnier (Byzantina Sorbonensia 5). Paris 1986, 123-187, hier 168-170 ( $\mathrm{N}^{\circ} 28$ - Alexis Paléologue), 176-178 ( $\mathrm{N}^{\circ} 32$ - Andronic Paléologue). F. Dölger in BZ 34 (1934) 126.

33 PLP VIII Nr. 21529.

${ }^{34}$ PLP VIII Nr. 21528.

35 Michael VIII. hat für Monembasia selbst ein Chrysobull hinsichtlich Abgabenbefreiung für den Handel in der Stadt erlassen (DölgER, Regesten III, Nr. 1897), das von Andronikos II. bestätigt wurde (DöLGER, Regesten IV, Nr. 2102); zu bestätigenden Urkunden des Michael IX. s. Dölger, Regesten IV, Nrr. 2617, 2626, 2628, 2642.

36 So Kalligas, Monemvasia 130.

${ }^{37}$ St. Binon, L'histoire et la légende de deux chrysobulles d'Andronic II en faveur de Monembasie. Macaire ou Phrantzès. EO 37 (1938) 274-311; DöLGER, Fälscher 373-376, $379-381$.

38 PLP VIII Nr. 21394.

39 PLP VIII Nr. 21347. 
wird auch durch ein anderes Detail annähernd gestützt: Alle drei Kaiser, die als Aussteller früherer Privilegien genannt sind, werden in P II als verstorben bezeichnet, was realiter erst nach 1331 zutraf, als Andronikos II. am 12./13. Februar hinschied.

Warum sollte Andronikos III. trotz all dem bereits wenige Jahre nach P I (1328) ein zweites, noch weiterreichendes Privileg für die Monembasioten konzediert haben? Eine plausible Antwort darauf liefert P II gleich eingangs (Z. 2-4): Die Begünstigten aus Pegai - lassen wir vorerst die Monembasioten selbst beiseite - halten sich gar nicht mehr in dieser ihrer

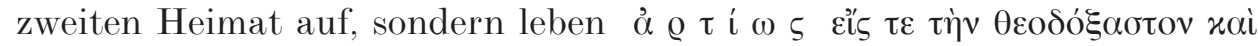

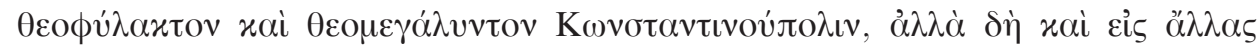

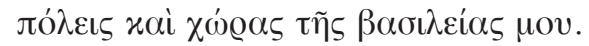

Ein langwieriger Prozeß vor dem patriarchalen Synodalgericht bestätigt 1356 diese neue Situation ${ }^{40}$. Im bereits zweiten Verfahren gegen den Metropoliten von Alania, Symeon, welches bald nach dem Amtsantritt des Patriarchen Philotheos Kokkinos im Herbst 1353 eröffnet worden war ${ }^{41}$,

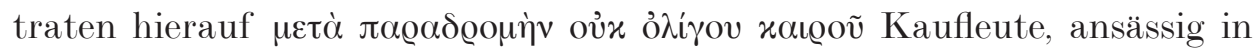

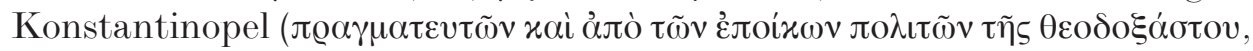

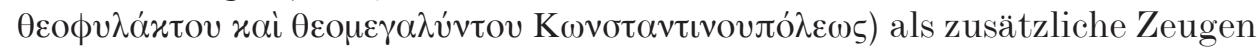
auf $^{42}$ (aufgrund des weiteren Prozeßverlaufs geschah dies im Frühjahr $\left.1354^{43}\right)$. Sie hatten die Verfehlungen des Metropoliten auf ihren Handelsfahrten im nördlichen Schwarzmeerraum selbst gesehen oder darüber gehört ${ }^{44}$. Da Symeon von einem ersten Verfahren unter Isidoros I. (1347-1351) in seinen Amtssitz zurückgekehrt war ${ }^{45}$, haben seine unter Kallistos I. untersuchten unkanonischen Handlungen 1351-1353 stattgefunden, die Anwesenheit der Kaufleute vor Ort in Tana fiel in jene Zeit (und möglicherweise schon zuvor). Namentlich von den Zeugen werden die Brüder Agape-

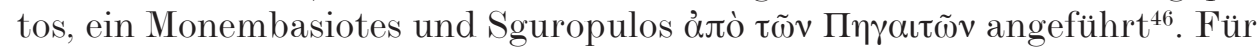

${ }^{40}$ Das Register des Patriarchats von Konstantinopel, 3. Teil: Edition und Übersetzung der Urkunden aus den Jahren 1350-1363, hrsg. von J. Koder - M. Hinterberger - O. Kresten (CFHB XIX/3). Wien 2001 (im folgenden PRK III), Nr. 215 (208-232).

${ }^{41}$ Zur causa insgesamt s. O. Kresten, Die Affäre des Metropoliten Symeon von Alania im Spiegel des Patriarchatsregisters von Konstantinopel. Anzeiger der Österreichischen Akademie der Wissenschaften, phil.-hist. Kl. 137 (2002) 5-40.

${ }^{42}$ PRK III Nr. 215, Z. 49-53 (214-216).

${ }^{43}$ Kresten, Affäre des Metropoliten Symeon 24-26.

${ }^{44}$ PRK III Nr. 219, Z. 56-57 (216). Matschke, Byzantinische Politiker 84-86.

45 J. Darrouzès, Les regestes des actes du patriarcat de Constantinople, vol. I: Les actes des patriarches, fasc. V: Les regestes de 1310 à 1376. Paris 1977, nr. 2308; PRK III Nr. 219, Z. 22-25 (214).

${ }^{46}$ PRK III Nr. 219, Z. 54-55 (216). 
andere Träger all dieser Namen sind mehrfach Geld- und Handelsgeschäfte belegt ${ }^{47}$. Obgleich in Konstantinopel wohnhaft, betonte Sguropoulos also seine Herkunft aus Pegai, mag sein, weil sie ihm (allein schon durch P I) Vorteile einbrachte ${ }^{48}$, mag sein, da die Übersiedlung rezent erfolgt war ${ }^{49}$, oder beides.

Eine neuerliche Emigration wird in den geänderten Machtverhältnissen begründet gewesen sein. Im osmanischen Raum besaß das kaiserliche Privileg keinen Wert mehr, der Handel über die Grenzen wird zudem diffizil zu bewerkstelligen gewesen $\operatorname{sein}^{50}$. Pegai war 1328/1329 noch byzantinisch ${ }^{51}$;

${ }^{47}$ Matschke, Byzantinische Politiker 84-85; Ders., Commerce 782 (ein Ioannes Agapetos 1310 in Kreta).

${ }^{48}$ Schon die geschäftliche Affinität mit einem Träger des herkunftsbestimmten Namens Monembasiotes (vgl. Matschke, Byzantinische Politiker 85), zudem das Gebiet seiner Aktivitäten spricht für Sguropulos als Angehörigen der Pegai-Monembasioten. Irgendwelche Indizien in Richtung einer Übertragung von deren Privileg auf alle aus Pegai emigrierten Bürger der Stadt (so P. Schreiner laut Matschke, Byzantinische Politiker 94, Anm. 70) vermag ich - außerhalb P II, das Schreiner aber als Fälschung beurteilt - nicht auszumachen.

${ }^{49}$ Es ist dabei nicht zu entscheiden (und darf auch vernachlässigt werden), ob seine Bezeichnung aus der Aktualität zu Zeiten des ohne Urteil abgebrochenen Verfahrens von 1354 (Darrouzès, Regestes V nr. 2369; vgl. PRK III Nr. 219, Z. 91-92, 104-111; Nikephoros Gregoras, Historia rhomaike XXXVII 7-8 [III 532-533 SchOPEN]) resultierte oder sein Wohnsitz erst bei der Neuaufnahme von 1356 die Grundlage bildete.

${ }_{50}$ Matschke, Commerce 775-776 zu entnehmen ist eine meiner Ansicht nach per analogiam charakteristische Entwicklung aus diesem Raum: Um 1350 bietet ein griechischer Händler Wein aus (dem Umland von) Trigleia (heute Zeytinbagi, $32 \mathrm{~km}$ nordwestlich von Bursa) an der kleinasiatischen Küste der Propontis) zu Konstantinopel einem venezianischen Käufer an (A. E. LAıou, Un notaire vénitien à Constantinople: Antonio Bresciano et le commerce international en 1350, in: Les Italiens à Byzance, ed. M. Balard, A. E. Laiou, C. Otten-Froux. Paris 1987, 79-151, hier 122). Ein bis zwei Jahrzehnte später hat das genuesische Handelsnetz mit seinen allseitigen Verträgen die Vermarktung dieses Produktes übernommen (M. BALARD, La Romanie génoise. XIIedébut du XVe siècle, I-II [Bibliothèque des écoles franc. d'Athènes et de Rome 235]. Rom 1978, II 844-845). Ergänzt sei, daß um 1325 dieser Wein gemeinsam mit dem aus Monembasia (Malvasier, s. oben Anm. 15) in Konstantinopel hochgeschätzt wurde (Ioannes Chumnos, Brief 6, ed. J. Fr. Borssonade, Anecdota nova. Paris 1844, 216).

51 Ioannes Kantakuzenos, Historiai II 5 (I 339-340 Schopen). Dölger, Regesten IV Nr. 2727. Der zu Pegai geschlossene Vertrag mit Tamerchanes / Demir Han (PLP XI Nr. 27425) sollte die westliche Flanke der Byzantiner bei der gegen Orhan, den Sohn Osmans, geplanten Militäraktion zur Entlastung Nikaias sein, die dann im Juni 1329 scheiterte (vgl. den Kommentar zu Ioannes Kantakuzenos, Geschichte II 6-8 in der deutschen Übersetzung von G. Fatouros und T. Krischer [Bibliothek der griechischen Literatur 21]. Stuttgart 1986, 176-182; M. DAS, Les relations byzantino-ottomanes au XIVe siècle. [Dissertation] Paris 1999, 120). 
erst 1354 hören wir wieder von der Siedlung, die jetzt türkischer Ausgangspunkt für Operationen in Thrakien ist ${ }^{52}$. Ein Privileg des Andronikos III., welches den Flüchtlingen einen Neustart in Konstantinopel und andernorts erleichterte, mußte vor Juni 1341 (Tod des Kaisers) ergangen sein. Letzteres Datum wäre zugleich terminus ante quem für die osmanische Eroberung von Pegai, durchaus passend zu den bedeutenden Orten an der südlichen Propontis (Prusa [Bursa], Lopadion [Ulubad], Nikaia [Iznik], Nikomedeia [Izmit]), welche ,zwischen 1325 und etwa 1340 in türkische Hand fielen" ${ }^{653}$.

Eine völlige Parallelität der Entwicklung in Bithynien und dem nördlichen Hellespont-Gebiet anzunehmen, läßt jedoch außer acht, daß Byzanz bis in die späten vierziger Jahre verschiedenen Gegnern gegenüberstand ${ }^{54}$, zum einen den Osmanen, welche, wie erwähnt, zu Lasten des Reiches expandierten, und zum anderen westlich des ab 1327 osmanischen Lopadion der Karasi-Dynastie mit Zentrum in Balıkesir ${ }^{55}$. Mit Tamerchanes / Demir Han aus deren Reihen hatte Andronikos III. zu Pegai 1328/1329 einen Vertrag auf Basis des territorialen status quo erreicht (s. bereits oben Anm.51). Aus der Historiographie ist das Datum nicht eindeutig zu ermitteln $^{56}$, aber die Parallelität mit dem Ergehen von P I im August 1328

${ }^{52}$ Ioannes Kantakuzenos, Historiai IV 38 (III 278 Schopen). G. Bogiatzes, H лoẃuฺ

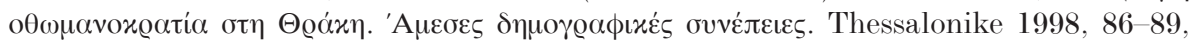
$97-100$.

53 Schreiner, Prostagma 204 mit Anm. 3.

${ }^{54}$ Grundsätzlich richtig erkannt bei Matschke, Byzantinische Politiker 84 mit Anm. 69.

${ }_{55}^{5}$ P. Schreiner, Die byzantinischen Kleinchroniken, 2. Teil: Historischer Kommentar (CFHB XII/2). Wien 1977, 232-233; X. De Planhol, Karasi. EI IV 652-653.

${ }^{56}$ Ioannes Kantakuzenos in seinem Geschichtswerk berichtet zur Alleinherrschaft des Andronikos III. zunächst über die ersten Auseinandersetzungen des Kaisers mit dem bulgarischen Zaren Michael III. Šišman (PLP Addenda zu Faszikel 1-8 Nr. 91377). Dessen Angriff wurde nicht ganz dreißig Tage seit der Machtergreifung von Andronikos III. (24. Mai 1328; Schreiner, Kleinchroniken II 234) bekannt (gemäß Nikephoros Gregoras, Historia rhomaike IX 8, 1 [I 430 Schopen]). Passend dazu vermerkt Kleinchronik 8 II, Notiz 20 (I 78 Schreiner), der Kaiser sei am 23. Juni aus der Hauptstadt wider Michael gezogen. Als sich das byzantinische Heer (wohl einige Tage später) Bizye (Viza) näherte, wich Michael auf bulgarisches Gebiet zurück. Nach einigen Tagen griffen die Byzantiner ihrerseits Diampolis an und plünderten es, ehe sie den Rückzug antraten (Io. Kant. II 3 [I 323-324 Schopen]; Soustal, Thrakien 116, 239), der etwa Mitte Juli stattgefunden haben muß. Danach ruhten über zwei Monate (!) lang die Feindseligkeiten. Kantakuzenos erwähnt dies nur kurz und fährt unter Beachtung dieses Erzählstranges mit den weiteren Ereignissen am bulgarischen Schauplatz fort. Michael eroberte (also frühestens gegen Ende September 1328) zunächst Bukelon (heute Fikla bzw. Matocina, $20 \mathrm{~km}$ nordnordwestlich Edirne) und begann hierauf mit der Belagerung von Probaton, heute Sinanköy, 21 km nordnordöstlich von Edirne (SoustaL, 
springt ins Auge. Selbige Urkunde wurde wahrscheinlich direkt zu Pegai ausgestellt $^{57}$, als der Kaiser dort wegen der Verhandlungen mit Tamerchanes / Demir Han weilte und durch deren erfolgreichen Abschluß der Region einige Friedensjahre verschaffte. Erst nachdem Orhan frühestens knapp vor 1350 auch das Karasi-Gebiet übernommen hatte ${ }^{58}$, geriet auch Pegai stark unter osmanischen Druck, dem es vor 1354 erlag.

Thrakien 222, 415-416). Andronikos III. zog ihm aus Didymoteichon über Adrianopel entgegen. Vor Probaton lagen die Heere einander auf einen Monat gegenüber (Io. Kant. II 3 [I 324 Schopes]). Verhandlungen führten dann alsbald zu einem Frieden (von den erwähnten Zeitabständen her im November 1328), man vereinbarte abschließend ein weiteres Treffen (Io. Kant. II 3 [I 324-329 Schopex]). Kantakuzenos geht nun auf andere Geschehnisse über (II 4-5 [I 329-339 Schopen]), die er wiederum von ihren Anfängen im Mai 1324 an darlegt (Begnadigung des Syrgiannes, deutsche Gesandtschaft, die noch an Andronikos II. gerichtet war; Aufstieg des Alexios Apokaukos). Der Aufenthalt des Andronikos III. nun zu Kyzikos, Artake/Erdek und in Pegai (Io. Kant. II 5 [I 339-340 Schopen]) ist vor dem zweiten Treffen mit dem Bulgarenzaren (II 5 [I340-341 Schopen) plaziert. Da unmittelbar der bithynische Feldzug von Mai 1329 nachfolgt (II 6-8, dazu s. oben Anm. 51), welcher zweifellos logistische Vorbereitungen erforderte, wird besagtes Treffen im März/April 1329 anzusetzen sein (DöLgER, Regesten IV Nr. 2723 ,ca. okt.“ vermengt die herbstliche Zusammenkunft mit dieser zweiten, vgl. bereits J.-L. Van Dieten, Nikephoros Gregoras, Rhomäische Geschichte [Bibliothek der griechischen Literatur 9]. Stuttgart 1979, 304). In Konsequenz daraus mochte Andronikos III. in Pegai entweder Ende 1328 / Anfang 1329 geweilt haben (ehe er nach Konstantinopel zurückkehrte) und dort den Vertrag mit Tamerchanes geschlossen haben (so Dölger, Regesten IV Nr. 2727) oder beides erfolgte bereits in den zwei Monaten (August / September 1328) zwischen dem ersten und zweiten Feldzug gegen Michael III. Šišman, was allerdings voraussetzt, daß Andronikos II. danach über Konstantinopel nach Didymoteichon zog und nicht den kürzeren Weg über die Dardanellen nahm.

${ }^{57}$ Ein explizites Beispiel für eine solche Ausfertigung seitens Andronikos III. auf Reisen ist ein „Chrysobull“, erlassen im August 1332 für Pietro de Canali (DölgER, Regesten IV Nr. 2785) in „Sperosa“ (Asperoza in der Bucht von Porto Lago, s. Soustal, Thrakien 184), worin es (laut lateinischer Kopie) heißt: „Et quia Imperium nostrum inventum fuit in loco distante de thesauro nostro, non habuimus bullam nostram auream consuetam ponendo in dictu sindicatu; tamen roboravimus manu nostra rubea subscriptione ..." (ed. G. M. Thомаs, Diplomatarium Veneto-Levantinum sive Acta et Diplomata res Venetas, Graecas atque Levantis illustrantia a. 1300-1350, I. Venedig 1880 [Reprint New York o.J.], 227). Zu einer weiteren, (wie bei P I) indirekt zu erschließenden Ausfertigung 1334 unterwegs s. O. Kresten, Zur Datierung, zum Schreiber und zum politischen Hintergrund dreier Urkunden des Kaisers Andronikos III. Palaiologos für das Serbenkloster Chilandariu. Anzeiger der phil.- hist. Kl. der Österreichischen Akademie der Wissenschaften 130 (1993) 67-99, hier 80-85, 90-92, 94-95 (Die Kenntnis beider Parallelbeispiele verdanke ich O. Kresten / Wien).

${ }^{58}$ C. Imber, The Ottoman Empire 1300-1481. Istanbul 1990, 19-22; DAs, Relations byzantino-ottomanes 121 datiert das Geschehen auf ca. 1345. 
Die kirchliche Situation zu Pegai bestätigt diesen historischen Verlauf. Vor dem Abkommen von 1328/1329 war die Metropolis von Pegai - bereits seit dem 13. Jahrhundert mit Parion (heute Kemer, $23 \mathrm{~km}$ westlich gelegen) in Personalunion vereint ${ }^{59}$ - in wirtschaftlichen Schwierigkeiten, was wohl in den türkischen Streifzügen begründet lag. Als Reaktion darauf wurde Pegai-Parion unter Metropolit Konstantin das thrakische Ganos (Gaziköy) als eigentliche Pfründe zugeteilt ${ }^{60}$. Im Rahmen der jährlichen Zuschüsse für das Patriarchat, die im September 1324 beschlossen wurden, lag Ganos mit 50 Hyperpyra im Mittelfeld ${ }^{61}$, wogegen Pegai und Parion keine Zahlungen zu leisten hatten; sie gehörten offenbar nicht zu jenen Kirchen, „die wohlhabend und durch ihre jeweiligen Vorräte dazu imstande“662 waren. Während die Verbindung mit Ganos bis spätestens 1329 dauerte $^{63}$, ist zunächst ungewiß, ob die Union von Pegai mit Parion fortbestand. Die zeitweilige Anwesenheit des Metropoliten von Pegai bei der konstantinopolitanischen Synode 1342 und im Herbst 1348 zeigt jedenfalls die weitere Existenz dieses Sitzes $a^{64}{ }^{6}$, Parion tritt hingegen allein nicht mehr auf. Zwei

${ }^{59}$ Einem Zusatz in Cod. F = Vat. gr. 1455, f. 223r-v der kirchlichen Rangliste Nr. 15 (ed. J. Darrouzès, Notitiae episcopatuum ecclesiae Constantinopolitanae. Paris 1981, hier 386, Z. 188-189, 192) zufolge wurde Pegai zwischen 1204 und 1261 zur Metropolis erhoben (und mit dem Erzbistum Parion vereint). Da die Stadt noch bis 1224 dauerhaft in lateinischer Hand war (Georgios Akropolites, Chronike syngraphe, c. 24 [I 38 HeISENBERG]), bildet dies einen terminus post quem dafür; auch ein Zusammenhang mit der Ansiedlung der Monembasioten ca. 1250 ist denkbar. Im Februar 1274 stimmte der Metropolit von Pegai und Parion der Kirchenunion zu, ein Schreiben von Papst Urban IV. rief im Juni jenes Jahres auch Pegai und Parion dazu auf, daran festzuhalten, ed. jeweils A. L. Tautu, Acta Urbani IV, Clementis IV Gregorii X (1261-1276). Città del Vaticano 1953, Nr. 42 (124) und Nr. 53 (140). Den unionsfeindlichen Tomos 1285 gegen Ioannes Bekkos unterzeichnete für Pegai und Parion dessen Metropolit Ignatios (V. LAURENT, Les signataires du second synode des Blakhernes. EO 26 [1927] 146).

${ }^{60}$ Dies erfolgte nach November/Februar 1318, als noch ein Metropolit allein von Pegai in einer synodalen Präsenzliste genannt wird, und dem 9. Mai 1324, zu welchem Datum diese Kombination erstmals belegt ist: H. Hunger - O. Kresten, Das Register des Patriarchats von Konstantinopel, 1. Teil: Edition und Übersetzung der Urkunden aus den Jahren 1315-1331 (CFHB XIX/1). Wien 1981 (im folgenden: PRK I), Nr. 61, Z. 33 (392) bzw. Nr. 73, Z. 8-9 (430).

${ }_{61}$ PRK I Nr. 88, Z. 72-73 (508). Darrouzès, Regestes V nr. 2119.

${ }^{62}$ PRK I Nr. 88, Z. 32-34 (506).

${ }^{63}$ Die Union ist zuletzt im Mai 1325 bezeugt (PRK I Nr. 89, Z. 7-8 [510]) und muß vor Dezember 1329 geendet haben, da zu diesem Zeitpunkt ein eigener Metropolit von Ganos, Menas, synodal präsent ist (PRK I Nr. 100, Z. 10 [564]).

${ }^{64}$ Das Register des Patriarchats von Konstantinopel, 2. Teil: Edition und Übersetzung der Urkunden aus den Jahren 1337-1350, hrsg. von H. Hunger - O. Kresten - E. Kislinger - C. Cupane (CFHB XIX/2). Wien 1995, Nr. 136, Z. 30 (290), Nr. 148, Z. 8 (384) und Nr. 151, Z. 117 (408). 
Synodalakte des Jahres 1351 unterzeichnete der Hypertimos Georgios je-

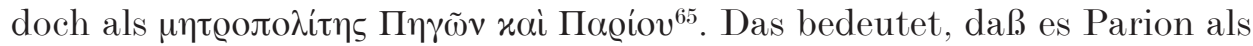
Bischofssitz faktisch nicht mehr gab und seine Zuständigkeit dauerhaft auf Pegai (gerade auch in der Titulatur) übergegangen war. Georgios dürfte jener Oberhirte gewesen sein, der nach August 1351 und vor August 1353 die Metropolis von Sozopolis (Sozopol) in Epidosis erhielt ${ }^{66}$, wegen der mißlichen Lage zu Pegai: „... die heiligste Metropolis Pegai war durch die entstandene außergewöhnliche Lage und durch den Angriff der Feinde mit der Duldung Gottes wegen der Fülle der Sünden fast vernichtet, sodaß sie nicht einmal mehr an ihren Resten zu erkennen war. Ihrem heiligsten Erzpriester, dem Hypertimos ... mangelte es selbst am Lebensnotwendigen, ja er verfügte nicht einmal über eine Unterkunft" ${ }^{* 67}$. Die zusätzliche Verleihung erfolgte vorerst nur provisorisch, aber bereits etwas vor Februar 1354 ersuchte der Oberhirte, ihm Sozopolis auf Dauer als Residenz zuzuweisen, und so geschah es ${ }^{68}$. Pegai war hiermit endgültig von der kirchlichen Autorität aufgegeben worden, ein mit den politischen Veränderungen um 1350 in dieser Region übereinstimmendes Geschehen.

Zu Zeiten von Andronikos III. (reg. 1328-1341) bestand für die PegaiMonembasioten hingegen noch keine Veranlassung, daß sie ihre bithynische Niederlassung aufgaben und Zuflucht in Konstantinopel und andernorts

${ }_{65}$ 1) PRK III, Nr. 179, Z. 13-14 (32): Georgios billigte im Jänner 1350 (auf Vorlage zusätzlicher Zeugenaussagen hin) nachträglich den Gerichtsentscheid über den Hieromonachos Niphon, welcher im September 1350 von der Anklage des Messalianismus freigesprochen worden war (dazu jüngst M. Hinterberger, Die Affäre um den Mönch Niphon „Skorpios“ und die Messalianismus-Vorwürfe gegen Kallistos I., in: La controversia palamitica, la chiesa e la teologia bizantina nel XIV secolo, a cura di A. RIGO [Orientalia venetiana 14]. Florenz 2003 [im Druck]). Ich halte es gleich Darrouzès, Regestes V nr. 2322 für denkbar, daß Georgios bereits am eigentlichen Verfahren 1350 teilgenommen hatte. - 2) E. Honigmann, Die Unterschriften des Tomos des Jahres 1351. BZ 47 (1954) 104-115, hier 106 (vgl. PG 151, 762A). Darrouzìs, Regestes V nr. 2326.

${ }^{66}$ PRK III Nr. 189. Der terminus post quem (so die Herausgeber von PRK III 90 mit Anm. 1) resultiert aus der Unterschrift eines eigenen Metropoliten von Sozopolis im Tomos von 1351 (Honigmans, Unterschriften 107), die dort freilich laut Darrouzès, Regestes V nr. 2326 hinzugefügt wurde, nach DAR Rouzès, Regestes V nr. 2357 ,a au plus tard en 1352". Das Ende des ersten Patriarchats von Kallistos I. im August 1353 als terminus ante quem (so PRK III loc. cit.) ist bereits von den zeitlichen Abständen her ganz logisch, aber derart nicht zwingend. Eine Formulierung wie hier PRK III Nr. 189

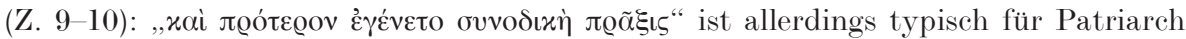
Philotheos, wenn er von Rechtsakten seines Vorgängers Kallistos I. spricht, dazu KreSTEN, Affäre des Metropoliten Symeon 6 mit Anm. 7.

${ }^{67}$ PRK III Nr. 189, Z. 1-8 (92).

${ }^{68}$ PRK III Nr. 189. Darrouzès, Regestes V nr. 2357. 
im byzantinischen Reich suchten. P II ist ergo trotz mancher Textaussagen (Vorgängerurkunden, Präsenz der Kaiserin), die vorerst auf Andronikos III. zu passen schienen, von der sachlichen Logik und dem historischen Umfeld nicht mit diesem Kaiser in Verbindung zu bringen. Auf ihn bezogen wäre es zudem sinnlos gewesen, die Gültigkeit des Privilegs auch auf Besitzungen, Domänen und Kastra zu betonen, welche dem Kaisersohn unterstünden (Z. 64-66), da zu seinen Lebzeiten keiner der beiden noch unmündigen Söhne, Ioannes (V.) (geboren 1332) (99 $^{6}$ und Michael (geboren $1337)^{70}$, eine solche Teilherrschaft innehatte ${ }^{71}$.

Ioannes $\mathrm{V}$. als Kaiser vermochte hingegen all den obigen Textaussagen von P II durchaus gerecht zu werden. Was die drei Vorgängerurkunden betraf, so haben Andronikos III. als Vater (mittels P I) und Andronikos II. als Urgroßvater (erwähnt in P I, Z. 2-3 [207 Schreiner]) für die PegaiMonembasioten nachweislich Privilegien erlassen, für Michael IX. gilt das bereits zuvor (211 mit Anm. 35) Gesagte. Die Kombination einer (im Vergleich zu P I) verstorbenen Kaiserinmutter, aber lebenden Gattin ist durch Johanna von Savoyen (s. Anm. 39) und Helene Palaiologina (Heirat 1347, verstorben 1397$)^{72}$ durch den Tod ersterer $1365 / 1366$ von da ab gegeben. Zwischen Frühjahr $1367^{73}$ und Sommer $1373^{74}$ übertrug der Kaiser seinem

69 PLP IX Nr. 21485.

70 PLP IX Nr. 21521.

71 Der argumentative Ansatz von Kalligas, Monemvasia 117-133, die von einer Datierung eines insgesamt echten Chrysobulls P II zwischen 1333 und 1341 ausgeht, verliert bereits hiermit seine Grundlage. Eine sogar noch präzisere Datierung auf November 1336 wird von ihr rein willkürlich erreicht, indem (op. cit. 129) im Jahresdatum (6825) $x$ für richtig $\mu$ verschrieben sein soll und ebenso grundlos die Indiktion von $\varepsilon^{\prime}$ auf $\varepsilon^{\prime}$ korrigiert wird. Zur Unhaltbarkeit des weiters von Kalligas behaupteten originären MonembasiaBezuges von P II (wogegen die Verbindung zu P I geringgeschätzt wird) s. unten $223-224$.

72 PLP IX Nr. 21365.

73 Terminus post quem ist die Rückkehr von Ioannes V. aus Ungarn bzw. Vidin zunächst nach Mesembria (Ankunft am 20. Jänner 1367) und dann in der Karwoche 1367 (11.-17. April) nach Konstantinopel, wobei auf dem Weg Sozopolis von Amadeus von Savoyen an den Kaiser übergeben wurde: SchreIner, Kleinchroniken II 296-297; Soustal, Thrakien 120-121, 455-456; O. Halecki, Un empereur de Byzance à Rome. Vingt ans de travail pour l'union des églises et pour la défense de l'empire d'Orient 1355-1375. Warschau 1930, 145-149; E. L. Cox, The Green Count of Savoy. Amadeus VI and Transalpine Savoy in the Fourteenth Century. Princeton 1967, 221-230.

74 Terminus ante quem ist der Versuch des Michael Palaiologos im November 1373, sich

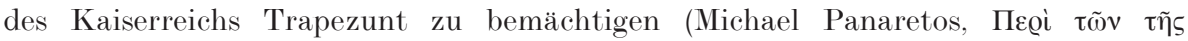

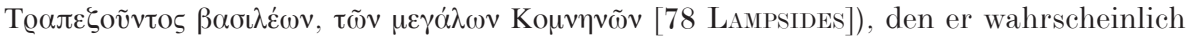
als Schwiegersohn des Dobrotica (PLP XII Nr. 29073) unternahm, der über Kalliakra (NO-Bulgarien) und (erst ab 1371?) die Gegend von Varna herrschte: P. SchreINER, 
dritten Sohn, dem Despoten Michael ${ }^{75}$, die Herrschaft über den am Schwarzen Meer gelegenen Küstenstreifen mit den Städten Mesembria (Nesebar), Anchialos (Pomorie), Sozopolis (Sozopol) und Agathopolis (Ahtopol) ${ }^{76}$.

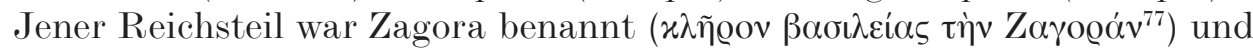
begegnet einem ebenso in einem der Zusätze von P II. Die Privilegempfänger sollen beim Ost-West-Transit auch frei sein von Abgaben, wenn sie

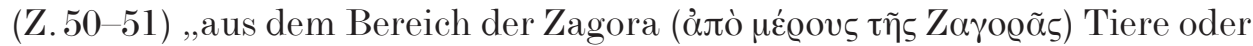
etwas anderes transportieren, sei es nach Sozopolis, nach Agathopolis oder nach Medeia (Midye)“. Gewiß differiert dieser Zagora-Begriff leicht von dem der Apanage von Michael, die allerdings in einem literarischen Kontext und daher nicht unbedingt präzise beschrieben wird. Zagor(i)a bezeichnete wohl primär das Hinterland der Küstenstädte ${ }^{78}$ und schloß bei einer gemeinsamen Oberhoheit diese dann ein. P II unterschied beide dennoch, vielleicht deshalb, weil die Abgaben in den Häfen eingehoben wurden.

Wie auch immer, erstmals harmonieren alle in P II gegenüber P I veränderten/ergänzten Urkundenelemente in ihren chronologischen Vorgaben miteinander, wenn von einer Texterstellung nach 1367 und (spätestens) vor dem Ende der Teilherrschaft des Michael $(1376 / 77)^{79}$ ausgegangen wird. Der zweite und endgültige Verlust im Herbst 1376 oder Frühjahr 1377 des P II (Z. 8) genannten ${ }^{80}$, zuvor erneut seit 1366 (dazu unten mit Anm. 91) byzantinischen Kallioupolis/Gallipoli (Gelibolu) an die Osmanen bestätigt den terminus ante quem. Offen bleibt dabei natürlich, ob nicht die genann-

Studien zu den BPAXEA XPONIKA (MBM 6). München 1967, 149-151; DERs., Kleinchroniken II 310-311; Soustal, Thrakien 121; V. GJuzelev, Ocerk varhu istorija na grad Nesebar v perioda 1352-1453. Godišnik na Sofiskija universitet. Filosofsko-istoričeski fakultet 64/3 (1970) 57-93, hier 63-66. Die Heirat (1371?) ist in Zusammenhang mit der Absicherung der Apanage gegenüber dem nördlichen Nachbarn zu sehen.

75 PLP IX Nr. 21522.

${ }^{76}$ Soustal, Thrakien 121: „,vermutlich im August 1369“ (ohne Begründung). V. GJuzelev, Chronicon Mesembriae. Belezki varhu istorijata na balgarskoto Cernomorije v perioda 1336-1448 g. Godišnik na Sofiskija universitet. Istoričeski fakultet 66/3 (1972/73 [ersch. 1975]) 147-192, hier 153-157.

77 G. Mercati, Notizie di Procoro e Demetrio Cidone, Manuele Caleca e Teodoro Meliteniota ed altri appunti per la storia della teologia e della letteratura bizantina del secolo XIV (StT 56). Vatikanstadt 1931, 129, Anm. 4 (Überschrift aus Cod. Urbin. 80, f. 166 ${ }^{\mathrm{v}}$ zum von Demetrios Kydones verfaßten Prooimion - dieses ed. nach Cod. Burn 75, f. $291^{\mathrm{v}}$ Sp. Lampros in BZ 5 [1896] 339-340 - des verleihenden Chrysobulls). Dölger, Regesten V Nr. 3222.

78 Soustal, Thrakien 503.

79 Schreiner, Kleinchroniken II 313-314.

${ }^{80}$ Schreiner, Kleinchroniken II 315-316; I. Beldiceanu-Steinherr, La conquête d'Andrinople par les Tures: La pénétration turque en Thrace et la valeur des chroniques ottomanes. TM 1 (1965) 439-461, hier 455, Anm. 101 
ten Städte an der Propontis einfach mechanisch aus P I übernommen wurden, ohne Bezug auf ihre aktuelle Handelsrelevanz: Rhaidestos speziell war schon ca. 1359/1360 temporär osmanisch geworden ${ }^{81}$. Umgekehrt kann mit dem jetzigen (vgl. P I, Z. 15 [207 Schreiner]) Fehlen von Ainos argumentiert werden, welches das türkische Vordringen im Marica/Hebros-Tal (1361 wird Didymoteichon ${ }^{82}, 1368 / 1369$ Adrianopel (Edirne) ${ }^{83}$ von ihnen erobert) von der Anbindung an die Routen nordostwärts gen Konstantinopel und zum Schwarzen Meer abgeschnitten hatte ${ }^{84}$. Der Fall 1367-1368 von Brysis (Pınarhisar) und Bizye (Viza) und 1373 Skopelos (Yoğuntaş / Polos) sowie Diampolis (Jambol) ${ }^{85}$ beeinträchtigte sicher fortan auch Aktivitäten in der Zagora-Region, weshalb einschränkend eine Genese des Wortlautes von P II bereits 1367-1373 (als Michael versuchte, alternativ in Trapezunt an die Macht zu kommen [dazu oben Anm. 74]) realistischer sein dürfte.

Aus dem Rahmen fällt auch so der Zeitraum von über eineinhalb Jahrzehnten, ehe man nach dem Verlust von Pegai (ca. 1351-1353) und der dadurch geänderten Situation an ein neues Privileg für die von dort Emigrierten heranging. Dieser Einwand setzt stillschweigend voraus, daß Pegai schon um 1354 gänzlich von der griechischen Einwohnerschaft, insbesondere der monembasiotischen Kolonie, verlassen worden war. Aber dem war nicht so, wie uns zufällig ein düsterer Lebensabschnitt des Gregorios Palamas enthüllt.

Der damalige Metropolit von Thessalonike war wenige Tage nach dem Erdbeben vom 2. März $1354^{86}$ von Tenedos auf dem Seeweg nach Konstantinopel aufgebrochen ${ }^{87}$. Widrige Winde zwangen das Schiff, nahe Gallipoli zu ankern. Dessen Mauern waren am 2. März eingestürzt, und die Stadt fiel

${ }^{81}$ Bogiatzes, Othomanokratia 111, 159. ODB III 1787.

82 Bogiatzes, Othomanokratia 113-115; Ph. A. Giannopoulos, Didymoteichon. Geschichte einer byzantinischen Festung. Köln 1975, 76-80.

${ }^{83}$ E. A. Zachariadu, The Conquest of Adrianople by the Turks. Studi veneziani 12 (1970) 211-217 (= DIEs., Romania and the Turks [c.1300-c.1500]. London 1985, Nr. XII); Beldiceanu-Steinherr, Conquête 452-458, 461; Bogiatzes, Othomanokratia 118-121; Schreiner, Kleinchroniken II 297-298.

${ }^{84}$ Soustal, Thrakien 132-146 mit Karte 133.

85 Bogiatzes, Othomanokratia 123-124, 127-128; Soustal, Thrakien 121, 220-221, 239, 446.

${ }^{86}$ Zum Datum s. Schreiner, Kleinchroniken II 283-284.

${ }^{87} \mathrm{Zu}$ den folgenden Geschehnissen s. den Bericht des Palamas selbst in einem Brief an seine Gemeinde, ed. A. Philippidis-Braat, La captivité de Palamas chez les Turcs: dossier et commentaire. TM 7 (1979) 109-221, hier 139-141, 145 (Text, 5-6, 10-11) und 196-197, 200 (Kommentar). Vgl. P. Charanis, On the Date of the Occupation of Gallipoli by the Turks. BSl 16 (1955) 113-117; Sp. VRyonis jr., The Decline of Medieval Hellenism in Asia Minor and the Process of Islamization from the Eleventh through the Fifteenth Century. Berkeley - Los Angeles - London 1971, 426-427. 
deshalb sogleich in die Hände der Osmanen; diese bemächtigen sich nun auch des Schiffes mit Palamas an Bord. Als Gefangener kam er zunächst nach Lampsakos und wurde nach Pegai getrieben. Erst nach einigen Tagen im Freien wies man ihm eine Kirche als Quartier zu, rund um die Laien und Mönche wohnten, welche sich der Gefangenen annahmen. Während der nächsten drei Monate genoß Palamas insbesondere die Gastfreundschaft des Maurozoumes. Die Familie Maurozo(u)mes hat ihre Wurzeln in der Peloponnes $^{88}$, und es darf vermutet werden, daß die Präsenz zu Pegai mit der monembasiotischen Handelskolonie ebendort zusammenhängt ${ }^{89}$, die also zumindest in Resten in den ersten Jahren der osmanischen Herrschaft weiterbestand.

Irgendwann nach 1354 und vor 1366 vermochte die christliche Gemeinde von Pegai sogar nochmals (unterstützt von dritter/byzantinischer Seite ?), die osmanische Oberhoheit abzuschütteln. Dies ist indirekt aus der Chronik des Derwisch Ahmed, genannt ${ }^{\mathrm{A}}$ Ašyqpaša-zade / ${ }^{\mathrm{A}} \mathrm{Ašik}$-Paša-Sohn (2. Hälfte des 15. Jahrhunderts), über die ,Denkwürdigkeiten und Zeitläufte des Hauses Osman“zu erschließen, welche über einen Feldzug von Murad I. berichtet. Auf die Nachricht über serbische Truppenbewegungen gegen Adrianopel hin zog der Osmanensultan selbst Streitkräfte in Kleinasien zusammen. ,Als er in die Gegend von Biga (= Pegai) kam, sagte er: ,... laßt uns diese Giauren hier unterwerfen. Dann wollen wir gegen jene anderen (= serbischen) Giauren ziehen‘... Er sandte nach Gelibolu (= Gallipoli) Nachricht. ,Schickt uns alle Schiffe her die ihr habt. Und auch die Schiffe aus Aydıncik (= Kyzikos) sollen herkommen‘. Es kamen also sehr viele Schiffe. ... Er ging zum Angriff über, und der Sieg wurde errungen. ... Die Kirchen wandelten sie zu Moscheen um, und die Häuser nahmen sich die Muslime zu ihren Wohnsitzen. "90 Das Geschehen wird von der Chronik auf das Jahr 766 der Hidschra datiert, was Oktober 1364 bis September 1365 christlicher Zeitrechnung entspricht.

Ein ganz sicherer terminus ante quem ist die Rückeroberung von Gallipoli zugunsten von Byzanz durch Graf Amadeo von Savoyen im August

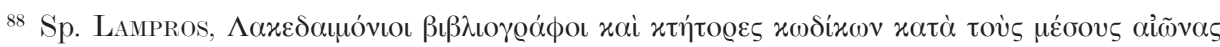

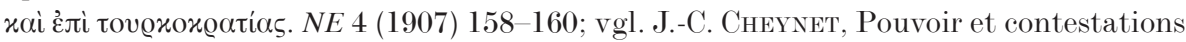
à Byzance (963-1210) (Byzantina Sorbonensia 9). Paris 1990, 146; PLP VII Nr. 17439 17444.

89 Byzantinische Forderungen auf Schadenersatz für Handelsverluste anno 1319 an die Venezianer betreffen auch einen gewissen „Maurosumi“ aus Monemvasia: Thomas, Diplomatarium I 72; LaIoU, Economy 206.

${ }_{90}$ Vom Hirtenzelt zur Hohen Pforte. Frühzeit und Aufstieg des Osmanenreiches nach der Chronik „Denkwürdigkeiten und Zeitläufte des Hauses Osman“ vom Derwisch Ahmed, genannt 'Asik-Pasa-Sohn. Übersetzt, eingeleitet und erklärt von R. F. Kreutel (Osmanische Geschichtsschreiber 3). Graz-Wien-Köln 1959, 85-86 (cap. 48). 
$1366^{91}$; für die folgenden zehn Jahre, bis Gallipoli 1376/77 dann endgültig an die Osmanen fiel ${ }^{92}$, vermochte Murad I. von dort keine Flottenhilfe anzufordern, wie von der Chronik erwähnt. Aktivitäten anno 1365 hingegen gegen Pegai (das übrigens schon 1354 als Sammelplatz für ein osmanisches Unternehmen gegen Gallipoli diente [s. oben 214 mit Anm. 52]) passen gut zur Hinwendung von Murad I. 1365/1366 zu den westlichen Teilen seines Herrschaftsgebietes, nachdem er die ersten drei Jahre nach dem Tod des Orhan (1362) beschäftigt gewesen war, seine Macht in Zentralanatolien zu stabilisieren ${ }^{93}$. Denkbar wäre es allerdings, daß 'Ašik-Paša-Sohn die serbische Niederlage an der Marica 1371 mit der Aktion gegen Pegai 1365 vermengt hat ${ }^{94}$, denn zum Zeitpunkt der Schlacht befand sich Murad I. eben in Pegai ${ }^{95}$, griff aber persönlich nicht ins Kampfgeschehen ein.

'Ašik-Paša-Sohn vermerkt zu Pegai abschließend, die Christen hätten „nach geraumer Zeit“ ein letztes Mal versucht, es zurückzuerobern; beim nächtlichen Angriff wäre die Stadt unter Greueltaten zerstört und dann ins Binnenland verlegt worden ${ }^{96}$. Egal ob die Pegai-Monembasioten diesen Vorstoß betrieben hatten oder nicht, das faktische Ende ihrer Kolonie ist schon zuvor mit 1364/65 anzusetzen; das einige Jahre später zu datierende Chrysobull kann daher nicht das Teilziel verfolgt haben, die Pegaiten nach Konstantinopel zu holen (so die an sich sinnvolle Überlegung von K.-P. Matschke [Brief vom 20. 7. 2002]). Die Aussage von P II über ihre zuvor

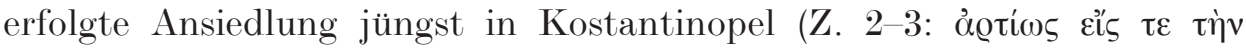

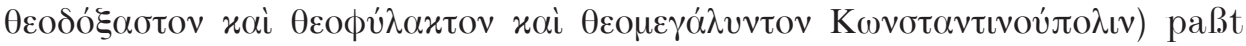
auch so durchaus zur neuen und zweiten Privilegerstellung 1367-1373, als neue Märkte offenzustehen schienen und die Hoffnung auf eine Rückkehr nach Pegai geschwunden sein mußte. Ein neues Chrysobull sollte zum einen die alten Rechte bestätigend auf die jetzt in Konstantinopel und anderen Städten lebenden Pegai-Monembasioten übertragen, zum anderen in der Hauptstadt und dem Zagora-Gebiet, dem nunmehrigen Zentrum wirt-

${ }^{91}$ Cox, Green Count 220, 235.

${ }_{92}$ Zur Datierung Beldiceanu-Steinherr, Conquête 455, Anm. 101; Schreiner, Kleinchroniken II 315-316.

93 Beldiceand-Steinherr, Conquête 442-446, 449.

94 Dieser Ansicht bereits J. von Hammer-Purgstall, Geschichte des osmanischen Reiches, I: Von der Gründung des Osmanischen Reiches bis zur Eroberung Constantinopels. 1300-1453. Pest 1827 (Reprint Graz 1963), 169-170, jedoch mit falscher Datierung auf 1363. N. Jorga, Geschichte des osmanischen Reiches, I. Gotha 1908, 240-241 setzt hingegen beide Ereignisse ins Jahr 1371.

${ }_{95}$ So Ahmed b. Lüțfullāh Müneğğimbaši, Gāmi’ ed-düvel, ins Türkische übersetzt durch A. Neīm. Constantinople 1868, III 293 (zitiert nach Beldiceanu-Steinherr, Conquête 443 und 451).

${ }_{96}$ Vom Hirtenzelt zur Hohen Pforte (wie Anm. 90) 86. 
schaftlicher Aktivitäten, zusätzliche Abgabensenkungen und -befreiungen (28 in P II gegenüber 13 in P I ${ }^{97}$ ) erteilen. Eine daraus resultierende Belebung des Handelsvolumens (und somit letztlich wiederum der Steuererträge) lag durchaus im staatlichen Interesse. Vielleicht förderte auch die Finanzkraft der Privilegempfänger die kaiserliche Bereitschaft, für diese Kreise ein Chrysobull zu erlassen. Es läßt sich sogar spekulieren, ob die Pegai-Monembasioten gar einen monetären Beitrag geleistet haben, um dem verschuldeten Ioannes V. im Frühjahr 1371 die Heimkehr aus Venedig zu ermöglichen. Dieser reizvolle, aber quellenmäßig unbewiesene Konnex (welchen P. Schreiner gesprächsweise anregte) würde die Entstehung von P II als Dankesgeste weiter auf 1371-1373 einengen.

Zwischen der Urfassung und dem uns überlieferten Text liegen jedenfalls rund zweihundert Jahre, in denen durch Abschreibfehler (ich erinnere

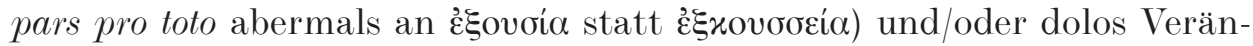
derungen am Original vorgenommen wurden. Als Hauptverantwortlicher ist unverändert (s. bereits oben 209-211) Makarios Melissenos anzusehen. Er wird es gewesen sein, welcher die Gültigkeit des Privilegs von den PegaiMonembasioten auf die Einwohner ihrer Mutterstadt Monembasia selbst manipulativ ausweitete.

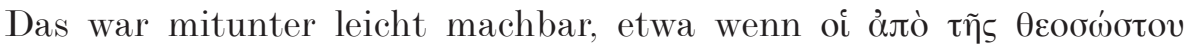

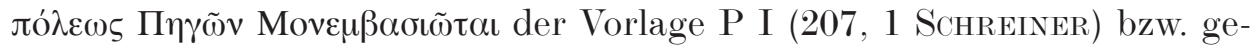

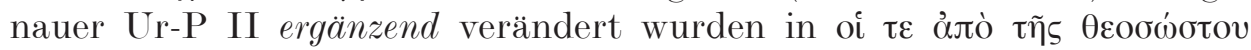

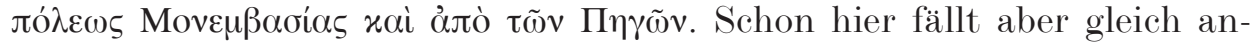
schließend auf, daß ja entgegen dem Wortlaut die Einwohner von Monembasia keineswegs jüngst nach Konstantinopel übergesiedelt sind, wie es im Urtext allein von der Pegai-Kolonie geheißen haben wird. Der Verfälscher

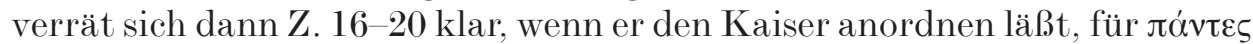

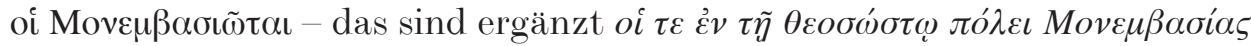

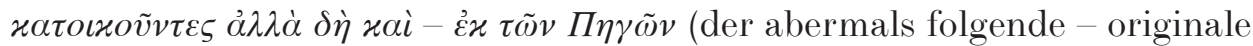
- Verweis auf die neue Bleibe in Konstantinopel betrifft hier allein sie)

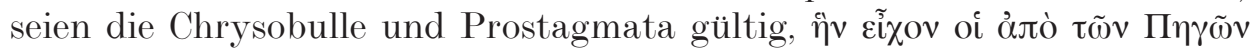

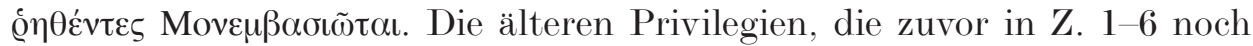
allen Monembasioten zugeschrieben worden waren, sind an dieser zweiten Stelle unverändert (und entlarvend) als solche ausgewiesen, welche sie wirklich waren, nämlich als allein für die Pegai-Monembasioten ergangene.

Die verfälschende Ausweitung des Prostagma-Inhalts P I nachträglich im Chrysobull P II auf alle Monembasioten ist im Fall der Abgabenreduktion zu Konstantinopel (P I, Z. 4-6; P II, Z. 7-9) überdies sinnlos. P II verfügt nämlich diesbezüglich nach der narrativen Bestätigung von P I

${ }_{97}$ Kalligas, Monemvasia 122. 
insgesamt (in Wahrheit zugunsten der Pegai-Monembasioten) dort ein neuerlich gesenktes Kommerkion von einem Goldstück (statt bisher zwei) pro hundert Nomismata (Z. 22-25). Auch die extra angeführten Jahrmärkte auf der Peloponnes offenbaren, wie bereits aufgezeigt (s. oben mit Anm. 23), lokalpatriotischen Übereifer des Verfälschers. Wer seiner Heimat zusätzliche Privilegien verschaffte, indem er sie zweihundert Jahre aus der Vergangenheit „bezog“, mußte wohl Vorsorge walten lassen, daß ob des großen Zeitabstandes keine Zweifel an der ungebrochenen Gültigkeit aufkamen. Das Chrysobull erließ der Kaiser daher angeblich auch zugunsten „der Kinder und Nachkommen, solange ihr Geschlecht andauert" (Z. 77-78). Es ist mit Matschke allerdings überlegenswert, ob nicht die ersten beiden Glieder doch original sind und die Rechtsansprüche der Nachfolgegenerationen sichern sollten.

Zusammenfassend ist zu P II und seiner Verfälschung festzuhalten, daß die 1367-1373 entstandene Urkunde(nvorlage) zugunsten der Pegai-Monembasioten späterhin manipulativ auf Monembasia ausgedehnt wurde, wobei noch falsche Datierungs- und Unterschriftselemente hinzutraten ${ }^{98}$. Offen bleibt, ob die Originalfassung von P II offiziell und rechtskäftig ausgefertigt wurde und das Fehlen der echten Datierungs- und Unterschriftsteile in der allein überlieferten Kopialform des Textes begründet liegt ${ }^{99}$. Oder kam P II über ein unterschriftsreifes Konzept nicht hinaus, bedingt durch die kaiserliche Abwesenheit 1369-1371 ${ }^{100}$ und den raschen geopolitischen Wandel, welcher die Privileginhalte bald obsolet werden ließ? So oder so besitzt das „Chrysobull“ P II als authentische, die historische Wirklichkeit widerspiegelnde Quelle aus dem späten 14. Jahrhundert seinen Wert.

${ }^{98}$ Vgl. bereits DöLger, Regesten IV Nr. 2383: „Man wird also auch hier damit zu rechnen haben, daß Makarios ein echtes stück in der hand gehabt und den text der von ihm zurechtgemachten geschichte des Sphrantzes ... einverleibt hat, nicht ohne retuschen leichterer art daran vorzunehmen; im ganzen macht der text des chrysobulls nämlich durchaus den eindruck der echtheit"; OıкоNomidès, Hommes d'affaires 88, Anm. 152: „Je me demande cependant si la source de Makarios Mélissènos n'était pas un chrysobulle perdu, concernant les privilèges de tous les Monembasiotes, auquel Mélissènos aurait fait des additions".

99 So Kalligas, Monemvasia 119, 130. Man vgl. aber aus dem 14. Jahrhundert mehrere vollständige „Empfängerkopien“ bei F. DöLger, Aus den Schatzkammern des Heiligen Berges. 115 Urkunden und 50 Urkundensiegel aus 10 Jahrhunderten. Textband. München 1948, Nr. 38, 40 (110-113, 115-116) bzw. in Actes de Kutlumus. Texte, ed. P. LEMERLE. Paris 1946, Nr. $11(60-64)$.

${ }^{100}$ PLP IX Nr. 21485; Halecki, Empereur 190-196, 227-232; A. A. Vasil’ev, Il viaggio dell'imperatore bizantino Giovanni V Paleologo in Italia (1369-1371) e l'unione di Roma del 1369. SBN 3 (1931) 151-193; J. W. BARKER, Manuel II Palaeologus (13911425): A Study in Late Byzantine Statemanship. New Brunswick, New Jersey 1969, 10-16; Schreiner, Kleinchroniken II 301-302. 


\section{Anhang: \\ Wiederabdruck des Chrysobulls P(egai) II \\ (Edition [mit Zeilenkommentar] von P. SchreIner in JÖB 27 [1978]}

$217-225)$

Kursiv gehaltene Textteile sind Ergänzungen/Veränderungen gegenüber der Privilegurkunde P I des Andronikos III. aus dem Jahre 1328 (ed. P. Schreiner in JÖB 27 [1978] 207-213). Da spätere Verfälschungen (von der Hand des Makarios Melissenos, dazu oben 209-211, 223) in P II nicht in jeder Einzelheit vollständig nachweisbar sind, erfolgt keine diesbezügliche Kennzeichnung.

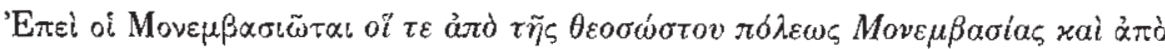

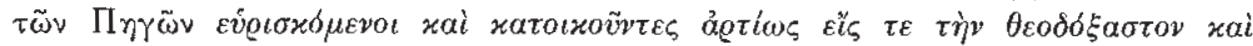

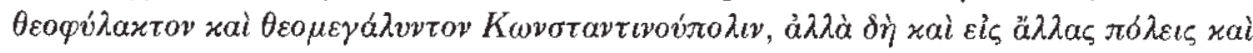

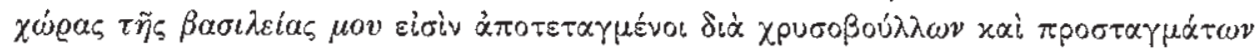

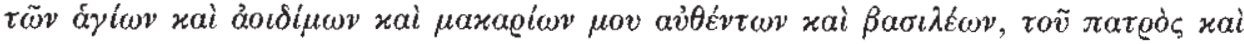

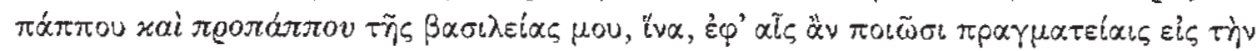

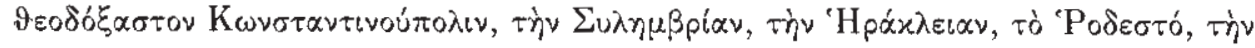

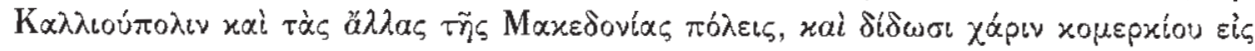

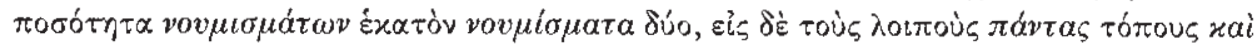

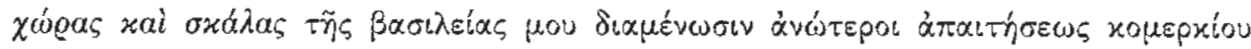

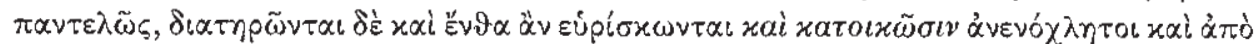

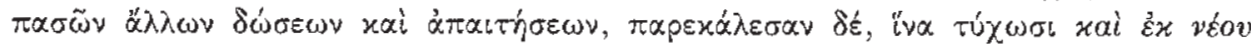

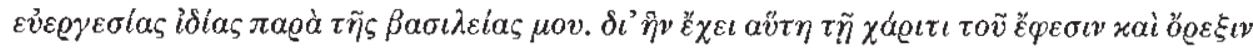

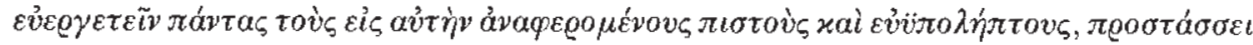

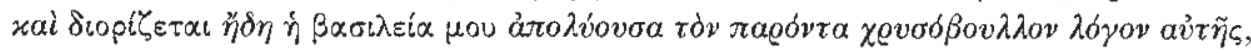

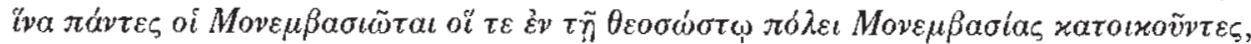

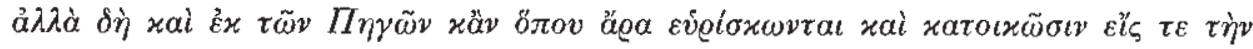

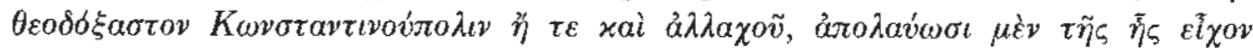

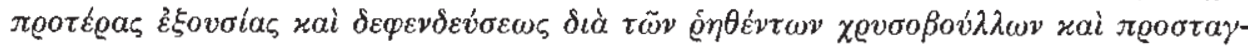

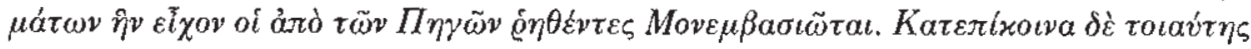

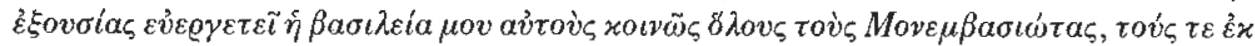

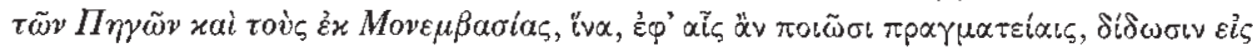

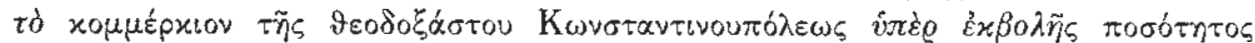

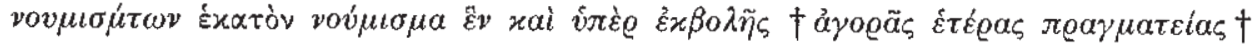

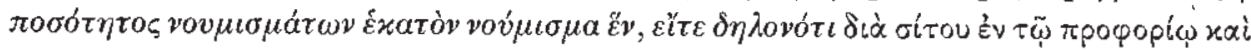

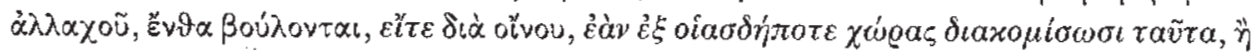
$A=\operatorname{cod}$. Ambr. P 23 sup
$\mathrm{B}=\operatorname{cod}$. Taurinensis B II 20
$\mathrm{P}=$ cod. Par. suppl. gr, 80

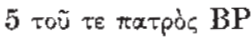
xai ${ }^{4}$ om. BP
11 ra! ${ }^{1}$ om. A

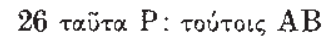




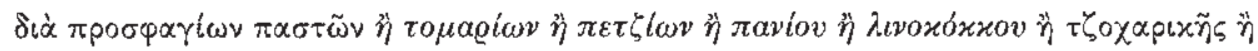

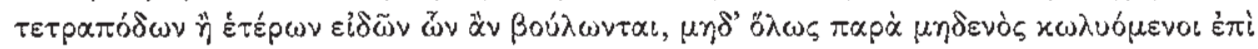

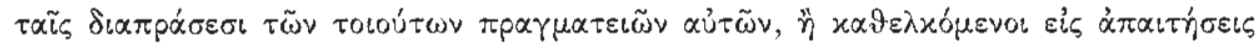

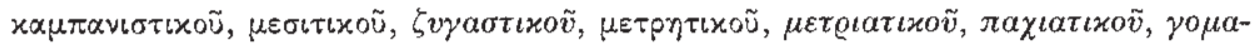

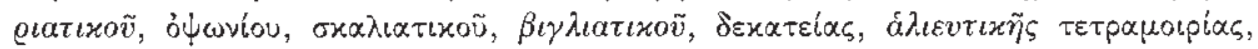

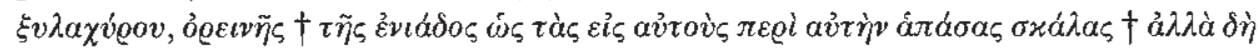

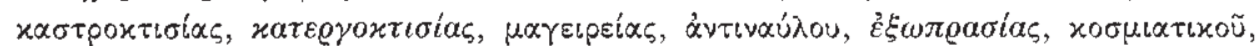

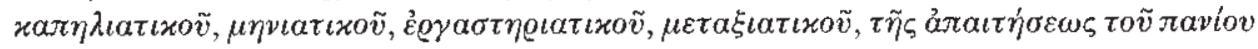

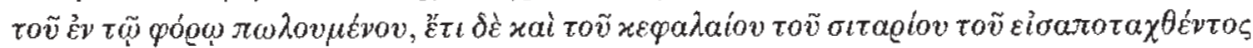

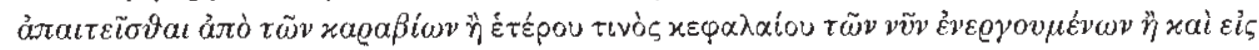

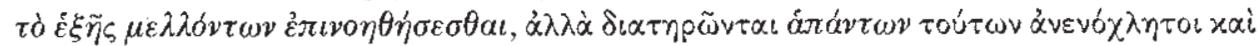

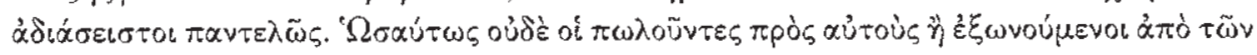

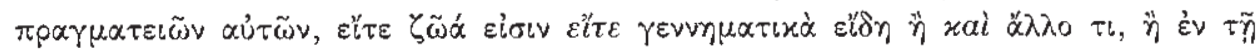

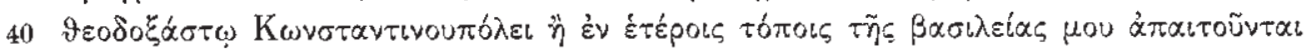

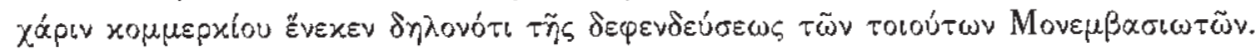

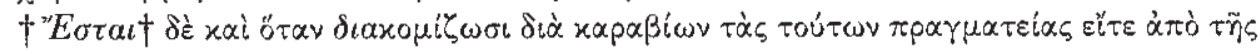

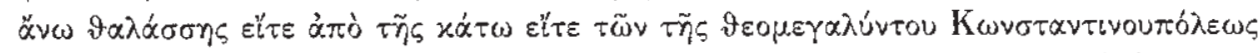

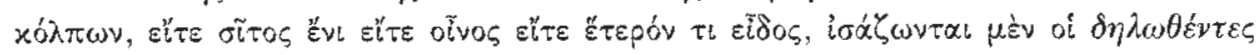

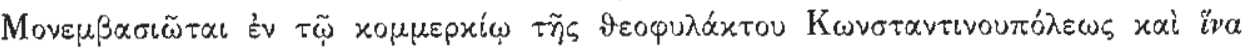

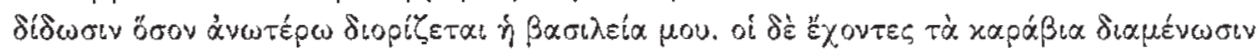

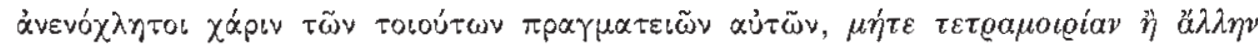

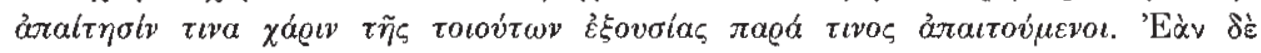

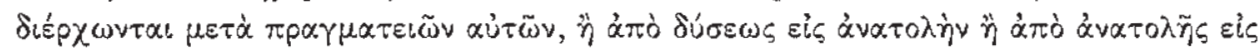

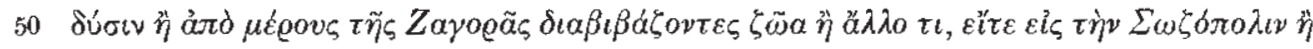

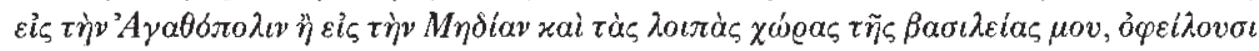

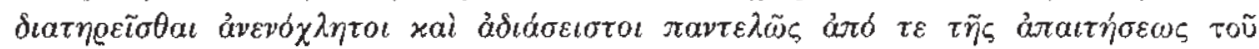

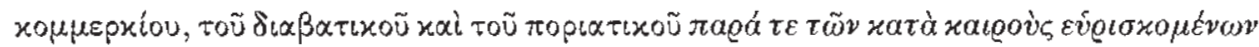

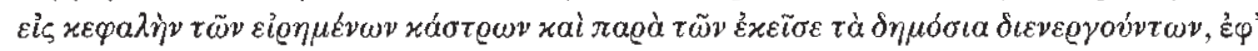

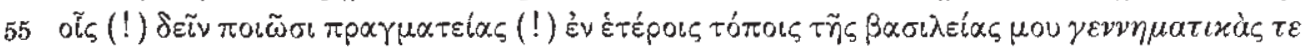

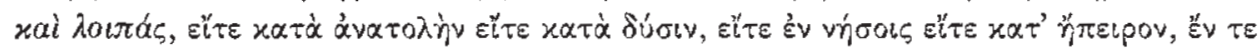

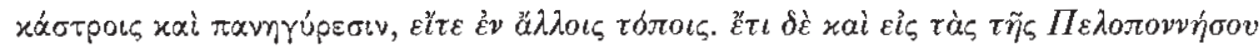

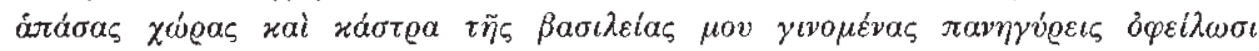

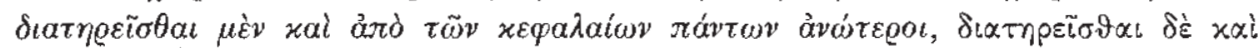

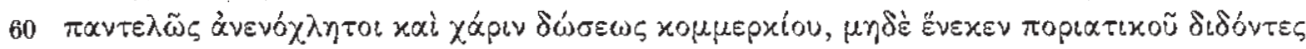

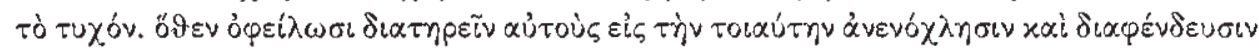

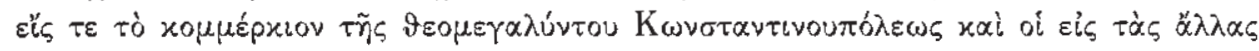

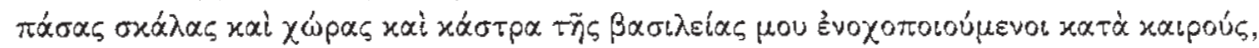

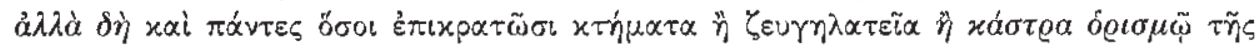




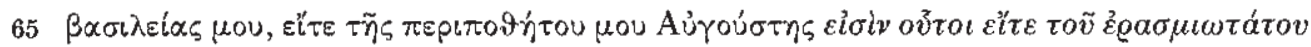

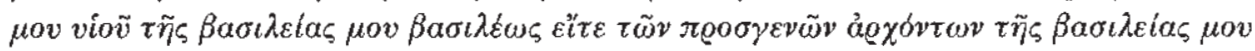

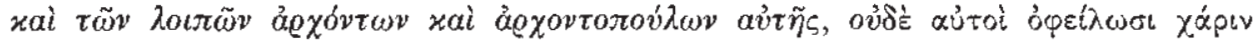

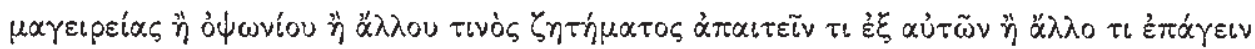

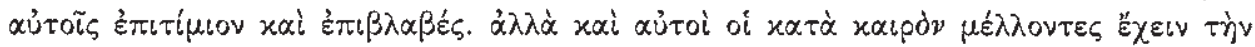

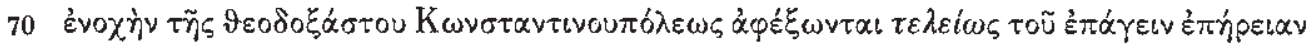

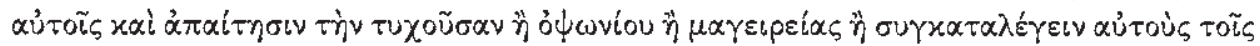

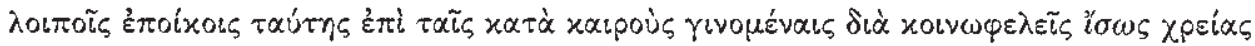

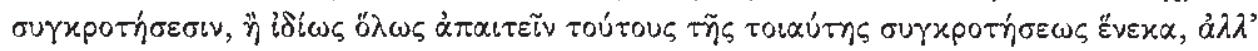

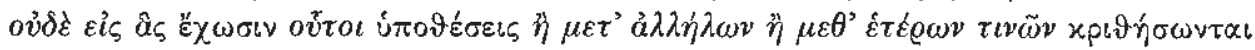

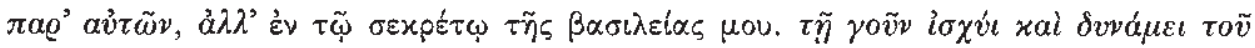

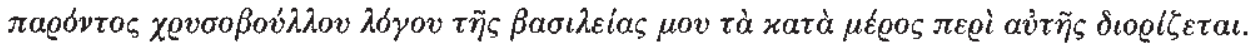

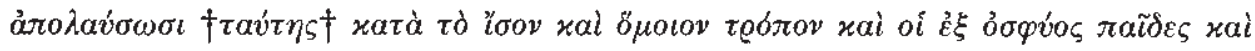

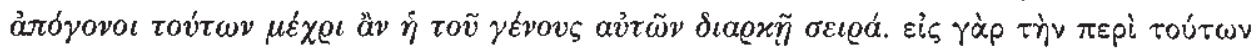

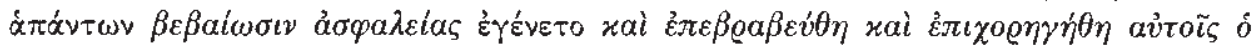

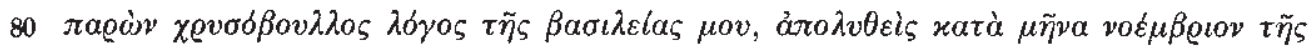

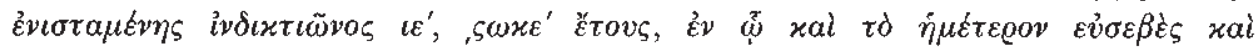

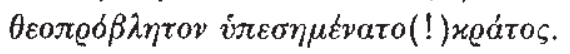

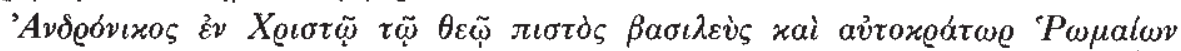

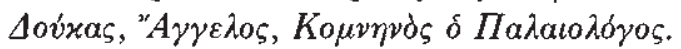

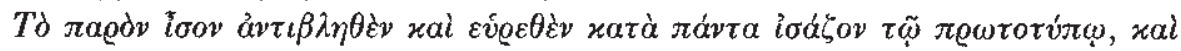
$\hat{\varepsilon} \gamma \varrho a ́ q \eta \delta i^{3} a ̉ \sigma \varphi a ́ d \varepsilon i \alpha \nu$.

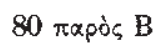


